\title{
High incidence of Plasmodium vivax malaria in newly arrived Eritrean refugees in Sweden since May 2014
}

K Sondén ${ }^{1}$, E Castro², L Trönnberg ${ }^{2}$, C Stenström³ ${ }^{3}$ A Tegnell ${ }^{2}$, A Färnert (anna.farnert@ki.se) $)^{1,4}$

1. Infectious Diseases Unit, Department of Medicine Solna, Karolinska Institutet, Solna, Sweden

2. The Public Health Agency of Sweden, Stockholm, Sweden

3. Department of Clinical Microbiology, Karolinska University Hospital, Solna, Sweden

4. Department of Infectious Diseases, Karolinska University Hospital, Solna, Sweden

Sondén K, Castro E, Trönnberg L, Stenström C, Tegnell A, Färnert A. High incidence of Plasmodium vivax malaria in newly arrived Eritrean refugees in Sweden since May 2014. Euro Surveill. 2014;19(35):pii=20890. Available online: http://www.eurosurveillance.org/ViewArticle. aspx?Articleld=20890

Since May 2014, an increase in Plasmodium vivax malaria has been observed in Sweden. As of 31 August 2014, 105 malaria cases have been reported in newly arrived Eritrean refugees, 84 of them $P$. vivax. The patients were mainly young men and reported migration through Ethiopia and/or Sudan. Severe anaemia and long symptom duration reflect inadequate healthcare during migration. Countries currently hosting Eritrean refugees need to consider $P$. vivax malaria in this group of migrants.

In Sweden, Plasmodium vivax accounts for 10 to $15 \%$ of the yearly ca 100 imported cases of malaria [1]. We report a dramatic increase in $P$. vivax cases among newly arrived Eritrean refugees, currently the third largest group of refugees in Sweden [2].

\section{Epidemiological hospital-based investigation}

$P$. vivax was diagnosed in seven newly arrived Eritrean refugees seeking care at Karolinska University Hospital in Stockholm in early June 2014. Information about this cluster and an inquiry about additional $P$. vivax cases were sent to the 28 infectious disease departments in Sweden. By 8 August 2014, physicians from 15 hospitals reported to have diagnosed $P$. vivax in 43 newly arrived Eritrean refugees since May 2014. No cases had been reported before May 2014. The patients were predominantly male (32/43) and aged 15 to 34 years (median 21) with $30 \%$ minors aged 15 to 17 years. All patients reported travelling from Eritrea through Ethiopia and/or Sudan to Libya and Italy before coming to Sweden between April and June 2014. Haemoglobin levels ranged between 40 and $136 \mathrm{~g} / \mathrm{L}$ (median: 110 $\mathrm{g} / \mathrm{L}$ ); four patients had haemoglobin levels lower than $70 \mathrm{~g} / \mathrm{L}$ and received blood transfusions. No other severe symptoms or deaths were reported. The diagnosis was established by conventional microscopy, and the patients were treated with chloroquine, except four patients who received artemether-lumefantrine (Riamet) due to initial uncertain species typing. All patients were prescribed relapse-preventing treatment with a 14-day course of primaquine, after excluding glucose-6-phosphate dehydrogenase deficiency.

\section{National surveillance data}

A marked increase in the number of notified malaria cases was also observed at the Public Health Agency of Sweden. This notification is based on mandatory reporting from physicians and diagnostic laboratories according to the Communicable Disease Act (Figure 1).

Until 31 August, 237 cases of malaria have been reported in Sweden in 2014 (102 P. vivax, 77 P. falciparum, $12 P$. ovale, two $P$. malariae, 44 unspecified species, and among those three mixed $P$. vivax and $P$. falciparum infections). According to the report from the notifying physician, 115 of them had been infected in Eritrea, Ethiopia and/or Sudan (72 P. vivax, seven $P$. falciparum, four $P$. ovale, 32 unspecified species and among those one mixed infection with $P$. vivax and $P$. falciparum). This corresponds to 9.0 $P$. vivax cases per month compared with 0.08 to 0.58 cases per month (between one and seven $P$. vivax cases annually) infected in those countries in the period 2010 to 2013 (Figure 2). There was no concomitant increase in $P$. falciparum cases.

By matching hospital and surveillance data, a total of 105 cases were confirmed to be Eritrean refugees (84 infected with $P$. vivax, five with $P$. falciparum, three with $P$. ovale and 13 with unspecified species). Some cases did not have the country of infection specified in the surveillance system, but were identified as Eritrean refugees in the complementary data provided by clinicians. Details on travel history, other than probable country, are usually not reported to the national surveillance system. Additional cases may therefore have occurred in this group, as well as among the cases where country of infection has not yet been reported. 
Number of malaria cases reported to the Public Health Agency, Sweden, 2010-14 (n=654)

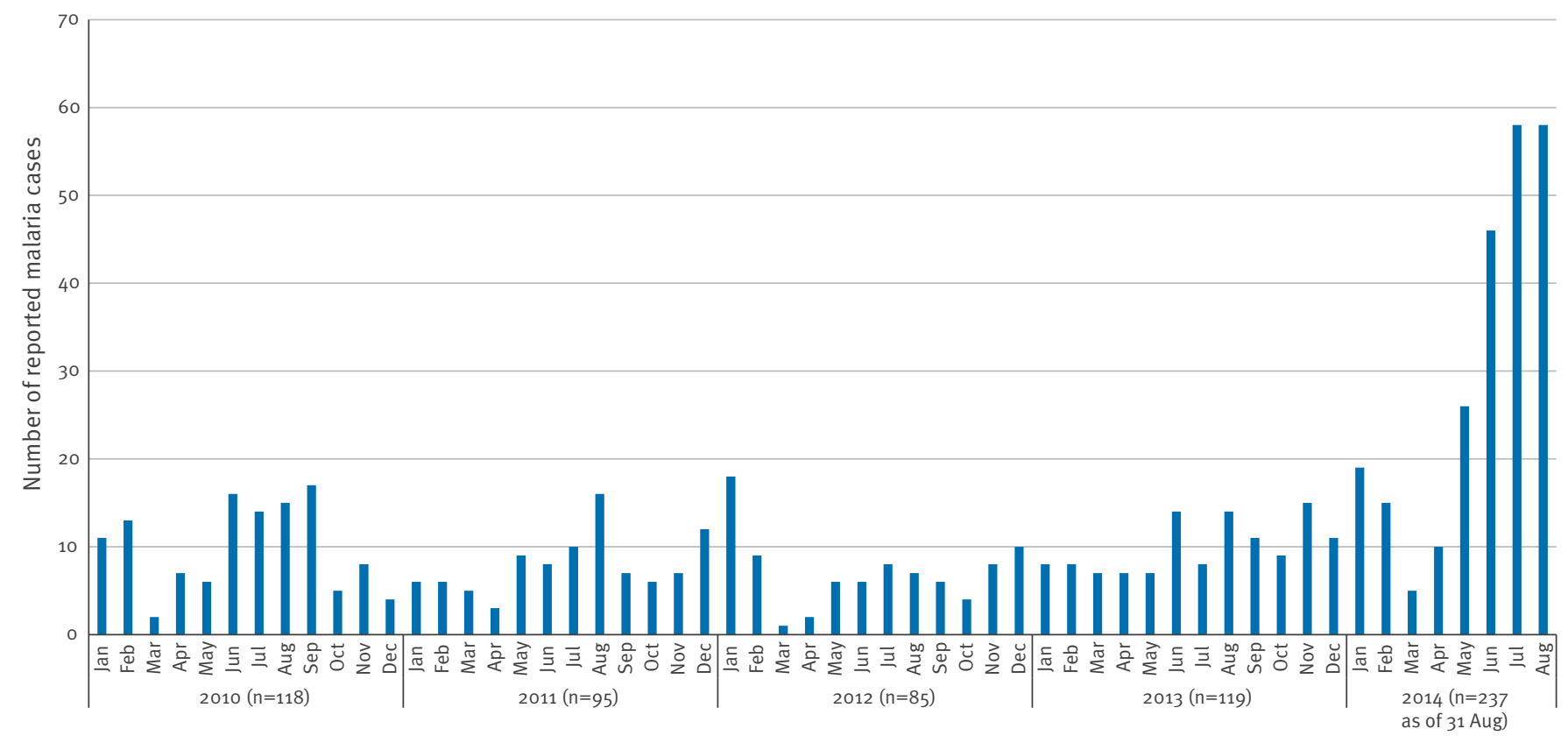

\section{Migration and estimated incidence}

According to the Swedish Migration Board [2], the annual number of Eritreans seeking asylum was 1,647 in 2011, 2356 in 2012, 4,844 in 2013, and 4,317 (708 minors) in 2014 until June, corresponding to an 1.8-fold increase in applications from 2013 to 2014. The estimated $P$. vivax incidence rate in 2014 was 19.5 per 1,000 Eritrean asylum seekers, and 38.2 per 1,00o Eritrean asylum seekers under 18 years of age. Detailed patient data were not available for 2012 and 2013; a crude estimate was therefore based on all $P$. vivax cases infected in Eritrea, Ethiopia and Sudan, generating an incidence rate of 1.2 in 2012 and 1.4 in 2013.

\section{Discussion}

This increase in $P$. vivax malaria cases clustered in newly arrived Eritrean refugees is the largest increase malaria cases diagnosed in Sweden since the reporting to the Swedish Public Health Agency was computerised in 1986. In Sweden, as in other European countries, $P$. vivax is predominantly diagnosed in travellers who have visited Asia or Oceania [3].

Although the number of asylum applications from Eritreans has increased in Sweden over the last years [2], the estimated $P$. vivax incidence was markedly higher in Eritreans who arrived in the past four months. The patients reported travelling through Ethiopia and/ or Sudan. We have not found any reports regarding ongoing outbreaks or markedly increased incidence of $P$. vivax malaria in those countries or in specific refugee camps over the past months. Nonetheless, our data suggest a high transmission of $P$. vivax along the route of migration of Eritrean refugees.
Also other European countries have observed $P$. vivax malaria in Eritrean refugees. In Oslo, Norway, 10 cases were reported to TropNetEurope in June 2014 [M. Jensenius, TropNet, personal communication, June 2014]. The Netherlands has also noted an increase in $P$. vivax cases in Eritreans, coinciding with an increased number of immigrants from that area [M. van der Sande National Institute for Public Health and the Environment, personal communication, July 2014]. A previous cluster of $P$. vivax in Eritrean refugees travelling through Sudan was described in Israel $2010[4,5]$.

$P$. vivax is endemic in more than 90 countries [6], with the highest risk in Central Asia [7]. In sub-Saharan Africa, the parasite is endemic mainly on the Horn of Africa and Madagascar [6], being geographically restricted by absence of Duffy antigens [8]. In Eritrea and Ethiopia, approximately $45 \%$ of malaria cases are due to $P$. vivax, compared with $5 \%$ in Sudan [6]. Eritrea reports a high rate of anti-malarial interventions and is considered to be on track to reduce malaria cases by more than $75 \%$ [6]. Malaria incidence had also decreased in Ethiopia over the last decade but there has been an increase in confirmed malaria cases including $P$. vivax in the past six years [6]. In Sudan, $P$. vivax transmission is limited and restricted to eastern and southern parts of the country [7], the areas where many refugee camps are located [9]. Libya and Italy are considered malaria-free [6]. Several patients described that they and many fellow migrants had symptoms of malaria during the journey; some were treated in Ethiopia and some in Sudan. Although it is difficult to determine the source geographically, it is likely that our patients acquired the infection in Sudan, Ethiopia or Eritrea. 


\section{FIGURE 2*}

Number of malaria cases, by origin of infection, reported per year to the Public Health Agency, Sweden 2010-14 $(\mathrm{n}=654)$

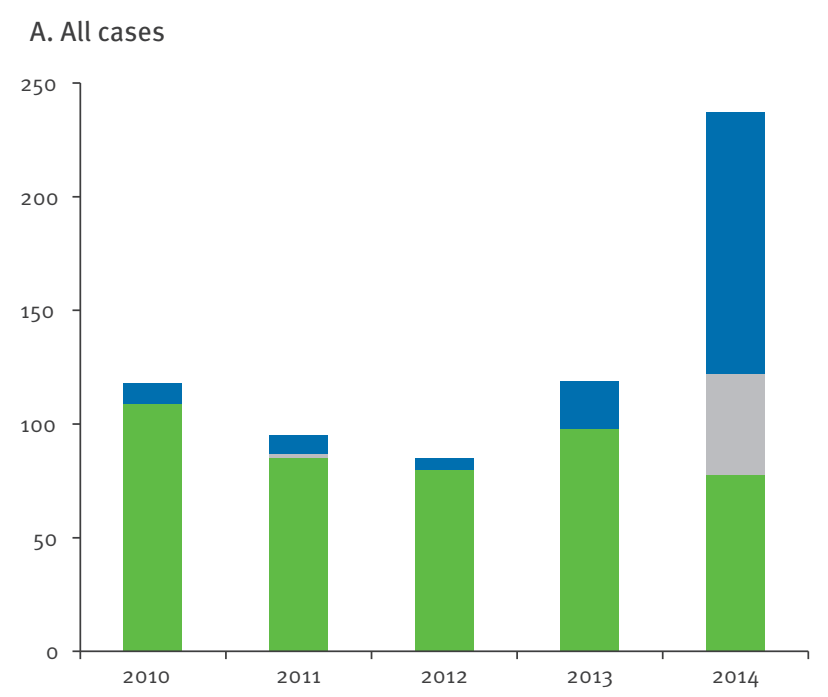

B. Plasmodium vivax

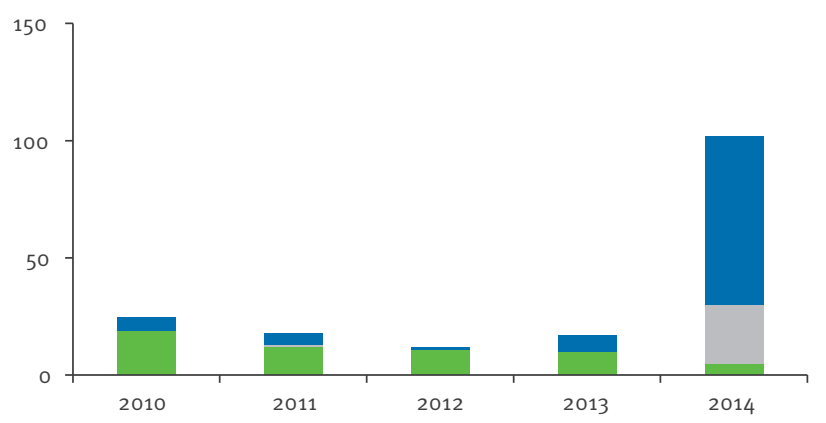

C. Plasmodium falciparum

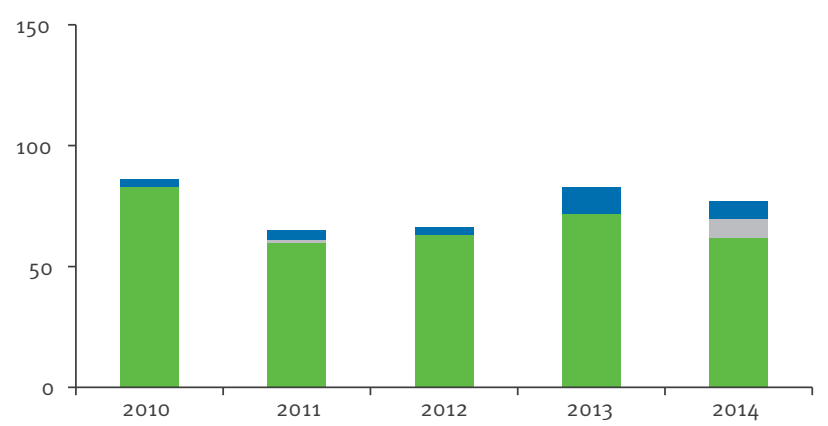

- Cases from Eritrea, Ethiopia and/or Sudan, reported as probable countries of infection for the newly arrived Eritrean refugees

Cases for whom country of infection has not yet been reported

Cases from other countries (in 2014: Afghanistan, India, Papua New Guinea, Peru and Tanzania)

Data for 2014 are as of 31 August.
Interestingly, although $P$. falciparum is the dominant species in those countries, the increase was specific for $P$. vivax. Geographical hotspots of transmission are likely to be situated along the route of migration, seeing as the $P$. vivax incidence among the refugees was higher than in Eritrea and Ethiopia (in 2012, one and seven per 1,000, respectively [6]). The high incidence, especially among minors, could also suggest a larger malaria-naïve population due to reduced endemicity in Eritrea. This should however also apply for $P$. falciparum, which may have been cleared by previous antimalarial treatments in some of the patients, resulting mainly in $P$. vivax relapses.

Several patients were anaemic and in need of blood transfusions. Malarial anaemia is well described for $P$. vivax, especially after long symptom duration and/or repeated episodes [10].

\section{Conclusions}

We report a high number of $P$. vivax malaria diagnosed in Sweden since May 2014. An increased incidence among newly arrived Eritrean refugees, especially in minors, indicates a change in exposure. Importantly, signs of long symptom duration during migration show the need for better healthcare for migrants and highlight the poor conditions that many refugees encounter during their escape. Countries currently hosting Eritrean refugees need to consider $P$. vivax malaria in these migrants.

\section{${ }^{*}$ Erratum}

The legend of Figure 2 was corrected on 5 September 2014. Blue and green were reversed.

\section{Acknowledgements}

We wish to thank all the Infectious diseases clinicians who reported on cases, the Swedish Migration Board for providing migration data; as well as Johan Ursing and Urban Hellgren for valuable comments on the manuscript.

Funding statement: Klara Sondén is supported by Karolinska Institutet and has a research/clinical internship at Karolinska University Hospital.

Conflict of interest

None declared.

Author contributions

Conceived the idea of the report: AF, AT, KS. Extracted and analysed data: LT, EC, AT, KS, CS, AF. Wrote the paper: KS, AT, AF. All authors have seen and approved the final manuscript. 


\section{References}

1. Sjukdomsstatistik: Malaria. [Disease statistics: malaria]. Solna: Folkhälsomyndigheten. [Accessed 8 Aug 2014]. Swedish. Available from: http://www.folkhalsomyndigheten. se/amnesomraden/statistik-och-undersokningar/ sjukdomsstatistik/malaria/? $\mathrm{t}=\mathrm{c}$

2. Applications for asylum received 1984-2013. . Migrationsverket. [Accessed 8 Aug 2014]. Available from:

http://www.migrationsverket.se/download/18.36084ac214622 cf6599137e/1403180720423/Tab+2+-+Application+for+asylum + received+1984-2013.pdf

3. Mühlberger N, Jelinek T, Gascon J, Probst M, Zoller T,

Schunk M, et al. Epidemiology and clinical features of vivax malaria imported to Europe: sentinel surveillance data from TropNetEurop. Malar J. 2004:3:5.

http://dx.doi.org/10.1186/1475-2875-3-5

4. Kopel E, Schwartz E, Amitai Z, Volovik I. Relapsing vivax malaria cluster in Eritrean refugees, Israel, June 2010. Euro Surveill. 2010;15(26): pii=19601.

5. Saidel-Odes L, Riesenberg K, Schlaeffer F, Smolyakov R, Kafka M, Borer A. Eritrean and Sudanese migrants presenting with malaria in Israel. Travel Med Infect Dis. 2011;9(6):303-5. http://dx.doi.org/10.1016/j.tmaid.2011.09.003

6. World Health Organization (WHO). World Malaria Report 2013. Geneva: WHO;2013. Available from: http://www.who.int/ malaria/publications/world_malaria_report_2013/en/.

7. Gething PW, Elyazar IR, Moyes CL, Smith DL, Battle KE, Guerra CA, et al. A long neglected world malaria map: Plasmodium vivax endemicity in 2010. PLoS Negl Trop Dis. 2012;6(9):e1814.

8. Miller LH, Mason SJ, Clyde DF, McGinniss MH. The resistance factor to Plasmodium vivax in blacks. The Duffy-blood-group genotype, FyFy. N Engl J Med. 1976;295(6):302-4. http://dx.doi.org/10.1056/NEJM197608052950602

9. United Nations High Commissioner for Refugees (UNHCR). Eritrea. 2014 UNHCR regional operations profile - East and Horn of Africa. Geneva: UNHCR. [Accessed 8 Aug 2014]. Available from: http://www.unhcr.org/pages/49e4838e6.html

10. Douglas NM, Anstey NM, Buffet PA, Poespoprodjo JR, Yeo TW, White NJ, et al. The anaemia of Plasmodium vivax malaria. Malar J. 2012;11:135. http://dx.doi.org/10.1186/1475-2875-11-135 